Design studies of the ATLAS muon Level-1 trigger based on the MDT detector for the LHC upgrade

This article has been downloaded from IOPscience. Please scroll down to see the full text article.

2010 JINST 5 C12021

(http://iopscience.iop.org/1748-0221/5/12/C12021)

View the table of contents for this issue, or go to the journal homepage for more

Download details:

IP Address: 137.138.124.142

The article was downloaded on 11/07/2011 at 10:24

Please note that terms and conditions apply. 


\title{
Design studies of the ATLAS muon Level-1 trigger based on the MDT detector for the LHC upgrade
}

\author{
O. Sasaki, ${ }^{a}, 1$ Y. Suzuki, ${ }^{a}$ K. Nagano, ${ }^{a}$ M. Ishino, ${ }^{a}$ M. Ikeno, ${ }^{a}$ K. Bendtz, ${ }^{b}$ T. Domae,${ }^{b}$ \\ H. Sakamoto, ${ }^{b}$ T. Kawamoto, ${ }^{b}$ C. Fukunaga,${ }^{c}$ H. Shichi, ${ }^{d}$ M. Tomoto, ${ }^{d}$ Y. Sugaya, ${ }^{e}$ \\ K. Tokunaga ${ }^{f}$ and H. Kurashige ${ }^{f}$ \\ ${ }^{a}$ High Energy Accelerator Research Organization (KEK), Institute of Particle and Nuclear Studies, \\ 1-1 Oho, Tsukuba-shi, Ibaraki-ken 305-0801, Japan \\ ${ }^{b}$ The University of Tokyo, International Center for Elementary Particle Physics and Department of Physics, \\ 7-3-1 Hongo, Bunkyo-ku, Tokyo 113-0033, Japan \\ ${ }^{c}$ Tokyo Metropolitan University, Graduate School of Science and Technology, \\ 1-1 Minami-Osawa, Hachioji, Tokyo 113-0033, Japan \\ ${ }^{d}$ Nagoya University, Graduate School of Science, \\ Furo-Cho, Chikusa-ku 464-8602, Japan \\ ${ }^{e}$ Osaka University, Graduate School of Science, \\ 1-1 Machikaneyama-machi, Toyonaka, Osaka 560-0043, Japan \\ ${ }^{f}$ Kobe University, Graduate School of Science, \\ 1-1 Rokkodai-cho, Nada-ku, Kobe 621-8522, Japan \\ E-mail: osamu.sasaki@kek.jp
}

ABSTRACT: The present muon Level-1 trigger of the ATLAS detector is given by dedicated detectors; RPC and TGC chambers in barrel and end-cap regions, respectively. The Monitored Drift Tube (MDT) chambers and the Cathode-Strip Chambers (CSC) are used for precision measurements of muon tracks. The performance of the muon Level-1 trigger is limited by the momentum resolution of the trigger chambers. In order to improve the trigger performance, a muon track finding scheme based on MDT signals is envisaged. Studies of the scheme and the algorithm are presented. The trigger latency is estimated to be approximately $3 \mu \mathrm{s}$.

KEYWORDS: Muon spectrometers; Trigger algorithms; Trigger detectors; Digital electronic circuits

\footnotetext{
${ }^{1}$ Corresponding author.
} 


\section{Contents}

1 Present system and improvement scenario 1

2 MDT based Level-1 Trigger 3

3 Latency estimation $\quad 5$

4 Conclusion 6

\section{Present system and improvement scenario}

The ATLAS muon spectrometer system consists of two types of chambers, which are for the purpose of either the precision momentum measurements of tracks or fast track finding for the Level-1 trigger, as shown in figure 1 [1]. The Monitored Drift Tube chambers (MDT, tube of $30 \mathrm{~mm} \phi$ ) and the Cathode-Strip Chambers (CSC) in the innermost end-cap region are used as precision-tracking chambers. The Resistive Plate Chambers (RPC) in the barrel and the Thin Gap Chambers (TGC) in the end-cap region are dedicated to triggering. The RPC has three stations at the middle (BML) and the outer (BOL) areas. The TGC consists of three stations at the middle area (EML). There is no trigger chamber at the inner stations (BIL, EIL and EEL). There is a toroidal magnetic field in

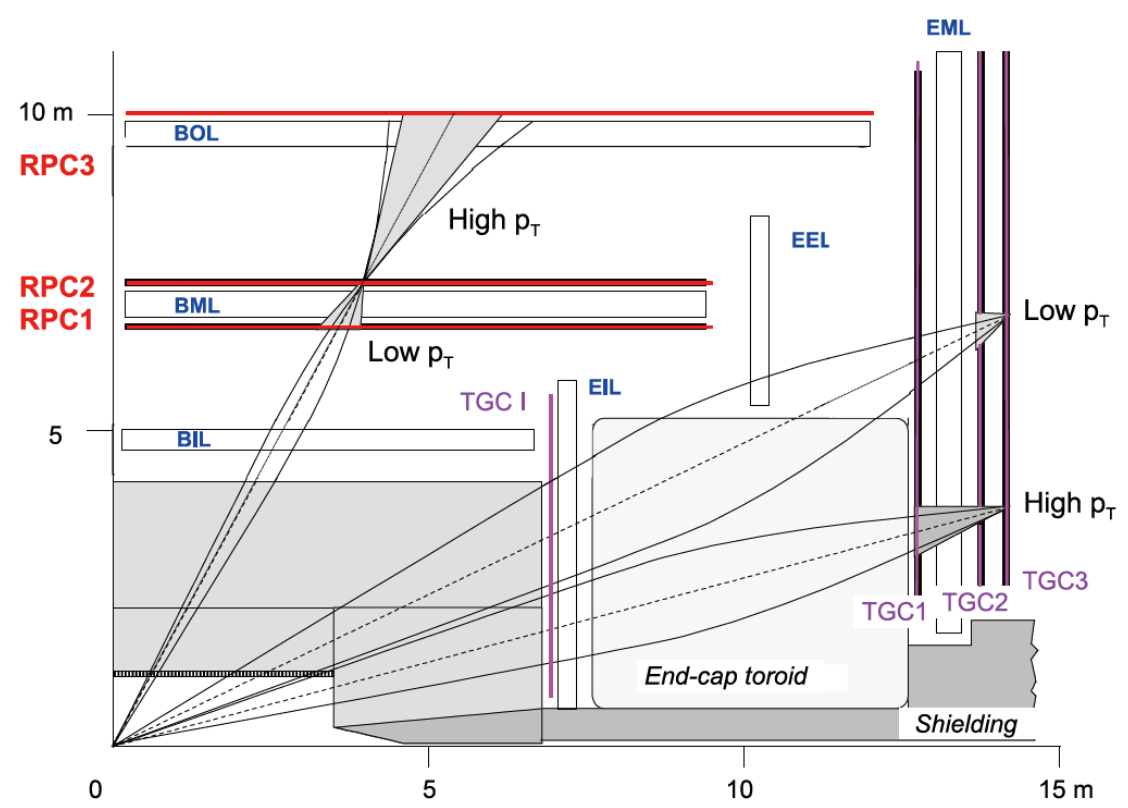

Figure 1. Cross-section of the present Muon Level-1 Trigger system. BIL/BML/BOL: Barrel Inner/Middle/Outer Large MDT. EIL/EEL/EML: End-cap Inner/Extra/Middle Large MDT. 

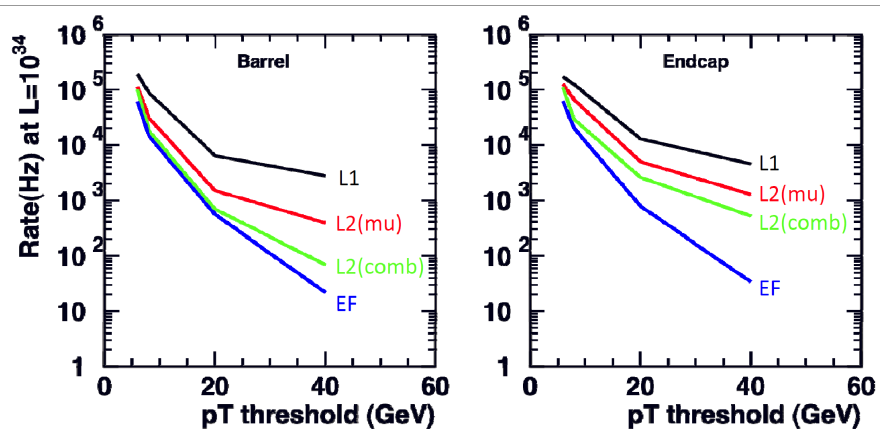

Figure 2. The simulated trigger rates of Level-1, Level-2 and Event Filter at L=1034 cm-2s-1. Black: Level1, Red: Level-2 (mu) using MDT data, Green: Level-2 (comb) combination with MDT and inner tracker, Blue: full tracking using all the data. The left is the barrel part and the right is the endcap part.
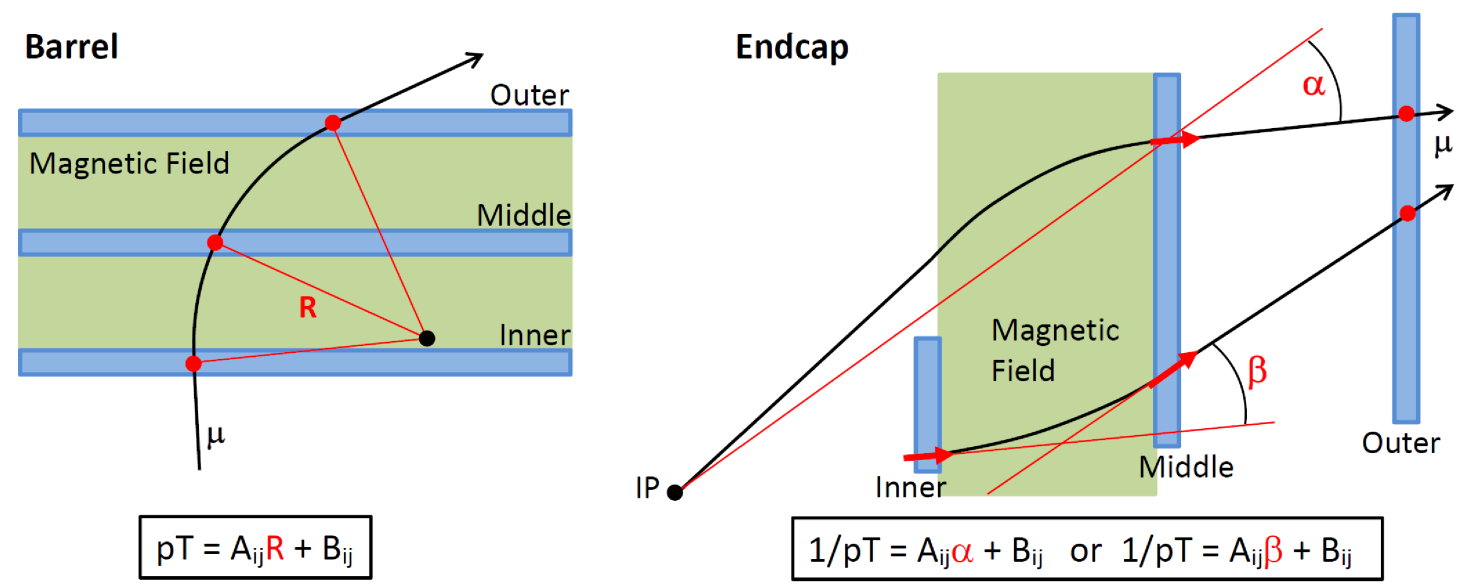

Figure 3. Level-2 Trigger Algorithm using MDT stations in Barrel and Endcap.

the barrel region, located between the inner and the outer station, as well as in the end-cap region between the inner and the middle station. The muon trigger system flags a muon track with the $p_{T}$ thresholds in the range of 6-35 GeV. The latency of the Level-1 trigger has been decided to be 2.5 $\mu \mathrm{s}$, which will be kept in the Phase-I upgrade.

Figure 2 shows the simulated trigger rates at Level-1, Level-2 and Event Filter (EF, Level-3) at $L=10^{34} \mathrm{~cm}^{-2} \mathrm{~s}^{-1}$ [2]. The trigger chambers and their trigger circuits satisfy the requirements of the $L=10^{34} \mathrm{~cm}^{-2} \mathrm{~s}^{-1}$ operation. In the luminosity upgrade of the LHC, improvements of the trigger performance are required in order to increase the robustness against background. According to simulation studies of the Level-1 muon trigger, the dominant contribution to the trigger rate is sharpness of the $p_{T}$ threshold curve, which is determined by the momentum resolution of the detector system. The long tail of the threshold curve towards the lower momentum region increases the background rate. The estimated momentum resolution of the present trigger system is $15-20 \%$ at $p_{T}=20 \mathrm{GeV}$ and $30 \%$ at $p_{T}=40 \mathrm{GeV}$. Detectors having higher momentum resolution for the trigger will make the $p_{T}$ threshold curves sharper. Additional trigger detectors at the innermost regions of the barrel and the end-cap will also improve the Level-1 trigger rate. 


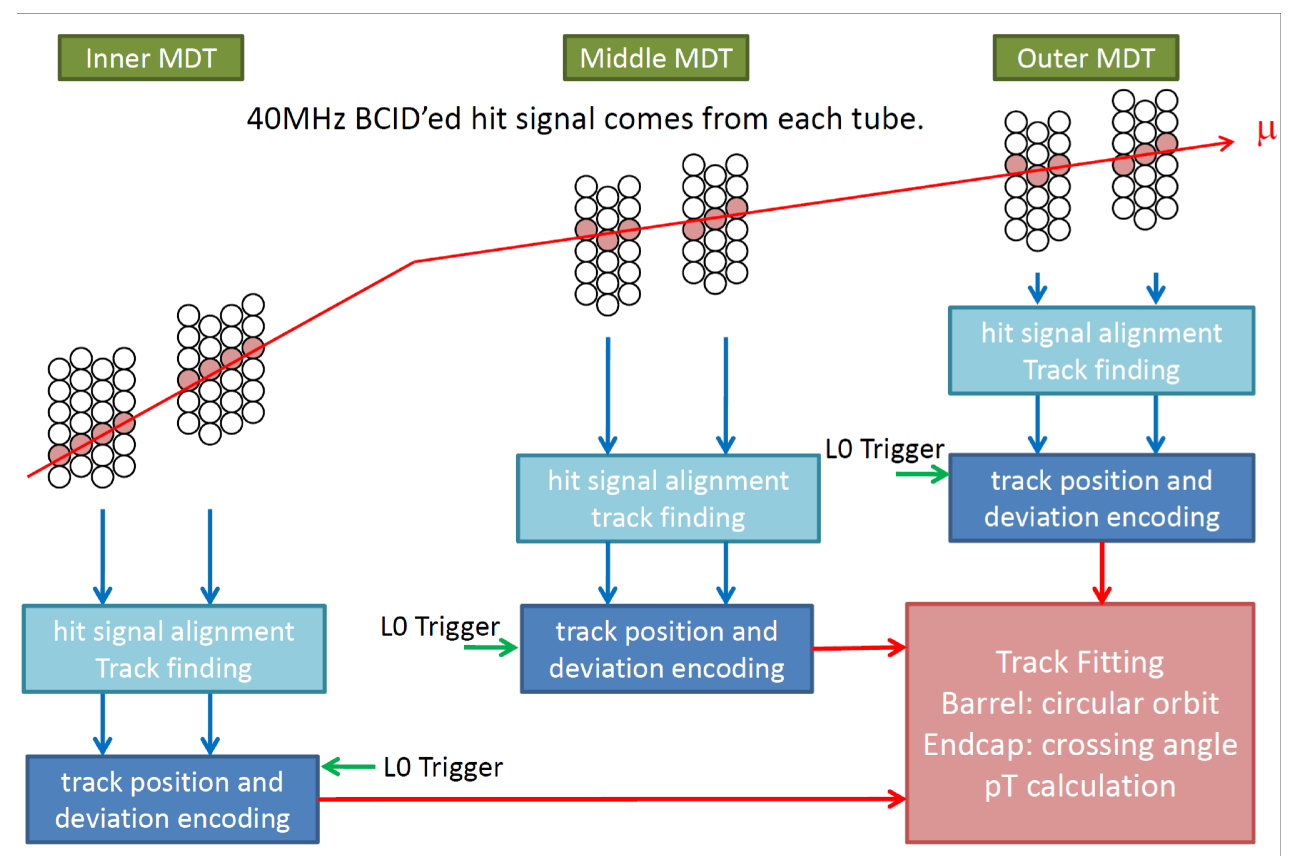

Figure 4. Block diagram of MDT based Level-1 Trigger.

A replacement of the existing trigger electronics would not be a solution to the problem, since it would not further reduce the muon trigger rate at low $p_{T}$ and accordingly the total rate. This is because the trigger performance is determined only by the spatial resolution of the trigger chambers and their geometrical layout. However, if we can implement the Level-2 (mu) algorithm using MDT data in the Level-1 hardware logic, a factor of $\sim 5$ reduction for the trigger rate can be expected. Figure 3 shows the algorithm of the Level-2 muon tracking (software code "muFast") [2]. Track fitting with a straight-line is made in each MDT station (Inner, Middle and Outer). In the barrel, the hit position at the center of each station is calculated. Fitting with a circular arc is made from three hit positions and then its radius $\mathrm{R}$ gives the $p_{T}$ value. In the end-cap, the crossing angle $\alpha$ between the track slope measured in Middle and the infinite momentum track from the interaction point (IP), or the angle difference $\beta$ between track slopes in Inner and Middle are measured. The angle $\alpha$ or $\beta$ is then converted to a $p_{T}$ value using Look-up-Tables (LUT).

\section{MDT based Level-1 Trigger}

A block diagram of the MDT based Level-1 Trigger is shown in figure 4. The signals from all the MDT tubes are bunch-identified (BCID) with the $40 \mathrm{MHz}$ LHC clock and sent to the counting room. Track fitting/finding with a straight line in each MDT station is made in parallel. The output from the finding logic is information about the track position and its deviation from an infinite $p_{T}$ track. The TGC and the RPC can provide unique bunch identification of a muon track. The bunch timing when a muon passes through MDT stations is obtained from the TGC or the RPC as a Level-0 signal. The Level-0 signal enables a bunch identification of the MDT system and will also assist in finally determining a track from the MDT signals. The track information from each station is combined and the $p_{T}$ value is calculated. 


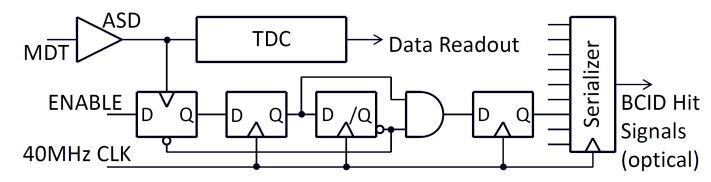

Figure 5. Bunch-Identification circuit.

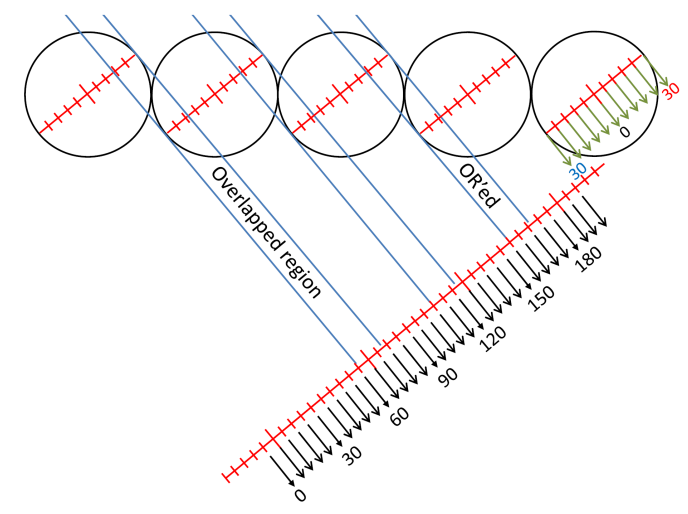

Figure 7. Spatial-aligned hit signals.

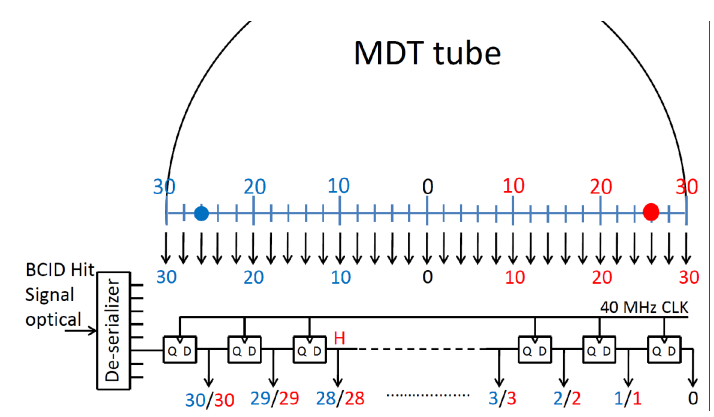

Figure 6. Hit signal decoding with 25 ns time slice using a 31-stage shift register.

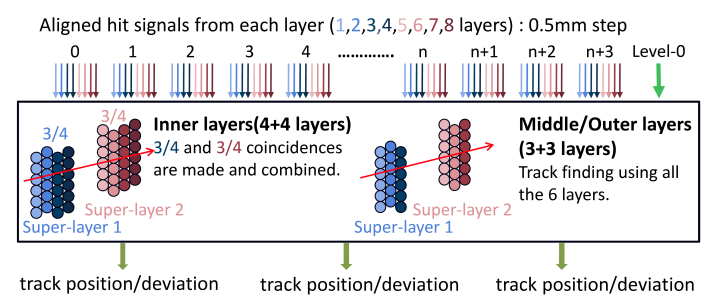

Figure 8. Station coincidence logic.

Figure 5 shows the BCID circuit, where leading edges of the hit signals from the ASD's are detected in each bunch $(25 \mathrm{~ns})$, which corresponds to $0.5 \mathrm{~mm}$ spatial resolution. The BCID hit signals are serialized and transmitted to the counting room via high speed optical links. The signals from the MDT front-end boards are deserialized and fed to decoding circuits as shown in figure 6. Each MDT tube has a 31-stage shift-register, which decodes the BCID hit signal to time/spatialaligned hit signals ( $25 \mathrm{~ns}$ timing and $0.5 \mathrm{~mm}$ spatial-aligned). One BCID hit signal is converted to 61 hit signals [30,29,28,,1,0,1,,28,29,30]. A "hit signal" is then transmitted from register to the next from $30 / 30$ to 0 at the $40 \mathrm{MHz}$ clock. The colours (blue or red) correspond to the two possible drift directions of the drift electrons. Decoded hit signals from each layer are projectively aligned in accordance with the expected incidence angle of an infinite $p_{T}$ muon, as shown in figure 7 . Hit signals from the overlapped region of neighboring tubes are logical-OR'ed. Aligned hit signals from each layer are fed to the track fitting/finding block in figure 8. The required $p_{T}$ threshold for the experiment at the LHC upgrade is larger than $20 \mathrm{GeV}$.

At the inner stations, the expected deviations of the tracks from infinite $p_{T}$ muons are less than $\pm 1^{\circ}$, so that one can restrict the track finding window to a very narrow range. 3-out-of-4 coincidence is imposed in each super-layer individually. The information of positions and track deviations from both super-layers are combined and the track candidates are extracted.

At the middle and outer stations, the track finding window is expanding into a larger area since the expected deviations of the tracks are up to $\pm 2.5^{\circ}$. We have to perform the track finding using 
Table 1. Latency estimation of MDT Track Trigger.

\begin{tabular}{|l|c|c|c|}
\hline & ns & CLK & Total CLK \\
\hline TOF from interaction point to MDT & 65 & 2.5 & 2.5 \\
\hline Propagation delay along wire & 30 & 1.5 & 4 \\
\hline MDT drift time (0-700 ns) & 700 & 28 & 32 \\
\hline ASD & 10 & 1 & 33 \\
\hline Bunch ID & & 2 & 35 \\
\hline Serializer + Optical Tx & & 2 & 37 \\
\hline Optical fiber cable (90m) & & 18 & 55 \\
\hline Optical Rx + De-serializer & & 3 & 58 \\
\hline Shift Register (28-steps, 0-700 ns) & & 0 & 58 \\
\hline Local coincidence & & 2 & 60 \\
\hline Track finding & & 2 & 62 \\
\hline Encoding & & 1 & 63 \\
\hline Track information from 3 MDT stations combined & & & \\
\hline Crossing angle calculation & & 3 & 66 \\
\hline pT calculation (LUT) & & 3 & 69 \\
\hline pT encoding & & 1 & 70 \\
\hline Cable to MUCTPI (10m) & & 2 & 72 \\
\hline MUCTPI & & 11 & 83 \\
\hline Cable to CTP (2.4m) & & 0.5 & 83.5 \\
\hline CTP & & 6 & 89.5 \\
\hline Cable to LTPI (10m) & & 2 & 91.5 \\
\hline LTPI + LTP + TTCvi + TTCex & & 2 & 93.5 \\
\hline Variable delay & & 2 & 95.5 \\
\hline Optical cable (110m max.) & & 22 & 117.5 \\
\hline TTCrq + fanout & & 3 & 120.5 \\
\hline Cable to frontend electronics & & 1.5 & 122 \\
\hline Total latency & & $3.05 \mu \mathrm{s}$ \\
\hline
\end{tabular}

aligned hit signals from all the 6 layers of the station in order to distill track candidates efficiently, shown in figure 8 . The algorithm for the track finding is being studied. The Level-0 trigger signal from TGC/RPC will be used to distill track candidates as well as the bunch identification. The information (position and deviation) of distilled tracks is sent to the next block, where the final track fitting is made using all the information from the three MDT stations. The fitting of a circular arc is made in the present Level-2 algorithm using the barrel data (figure 3: Barrel). It may be too ambitious to implement a similar algorithm in hardware within the limited latency budget and studies for the algorithm of hardware are to be continued. The implementation of the algorithm for the end-cap part is feasible for hardware. Using the fitting results, $p_{T}$ values are obtained. An algorithm based on LUT's is envisaged.

\section{Latency estimation}

Table 1 shows the estimated latency of the MDT muon track trigger. The latency budget from "Cable to MUCTPI" to "Cable to front-end electronics" in the table is the measured values of 
the present TGC Level-1 system. The total latency is $3.05 \mu \mathrm{s}$, which is $500 \mathrm{~ns}$ longer than that of the present TGC system. A considerable contribution comes from the MDT drift time. The contingency of the present ATLAS system is estimated to be less than 20 clocks. Further effort to reduce the latency of the Level-1 trigger and improvement of each sub-detector to cope with longer latency are desired for the upgrade of the LHC.

\section{Conclusion}

The ATLAS muon Level-1 trigger based on the MDT signals is being studied for the LHC upgrade. The conceptual design has been proposed and we have started R\&D towards a concrete design for implementation. The latency of the trigger is estimated to be approximately $3 \mu \mathrm{s}$. The software code for the trigger simulation is being developed to study the algorithm of the track fitting/finding and the $p_{T}$ calculation. The algorithm should be hardware-oriented to be able to implement in FPGA's and is better to be fully synchronized logic with fixed latency ( $<3.2 \mu \mathrm{s}$ in total). We also have to evaluate the tracking efficiency, any efficiency holes and the probability of incorrect tacking using real data as well as Monte Carlo data.

\section{References}

[1] G. Aad et al., The ATLAS Experiment at the CERN Large Hadron Collider, 2008 JINST 3 S08003.

[2] G. Asd et al., Expected Performance of the ATLAS Experiment: Detector, Trigger and Physics, Rev. Sci. Instrum 76 (2005) 054503 [arXiv : $0901.0512 v 4]$. 